\title{
SOD2- and NRF2-associated Gene Signature to Predict Radioresistance in Head and Neck Cancer
}

\author{
JOO KYUNG NOH ${ }^{1}$, SEON RANG WOO ${ }^{2}$, MIYONG YUN ${ }^{3}$, MIN KYEONG LEE ${ }^{1}$, MOONKYOO KONG $^{4}$, \\ SOONKI MIN ${ }^{4}$, SU IL KIM ${ }^{2}$, YOUNG CHAN LEE ${ }^{2}$, YOUNG-GYU EUN ${ }^{1,2}$ and SEONG-GYU KO ${ }^{5}$ \\ ${ }^{1}$ Department of Biomedical Science and Technology, Graduate School, \\ Kyung Hee University, Seoul, Republic of Korea; \\ ${ }^{2}$ Department of Otolaryngology-Head and Neck Surgery, \\ Kyung Hee University Medical Center, Seoul, Republic of Korea; \\ ${ }^{3}$ Department of Bioindustry and Bioresource Engineering, \\ College of Life Sciences, Sejong University, Seoul, Republic of Korea; \\ ${ }^{4}$ Department of Radiation Oncology, Kyung Hee University Medical Center, Seoul, Republic of Korea; \\ ${ }^{5}$ Department of Preventive Medicine, College of Korean Medicine, Kyung Hee University, Seoul, Republic of Korea
}

\begin{abstract}
Background: We propose a novel prognostic biomarker-based strategy for increasing the efficacy of radiotherapy $(R T)$ in head and neck squamous cell carcinoma (HNSCC). Materials and Methods: We identified genes associated with superoxide dismutase 2 (SOD2) and nuclear factor erythroid-2-related factor 2 (NRF2) from gene-expression data of The Cancer Genome Atlas (TCGA) by calculating Pearson correlation. Patients were divided into two groups using hierarchical clustering. Colonyformation assay was performed to determine radioresistance in HNSCC cell line CAL27. Pathway analysis was conducted using The Database for Annotation, Visualization and Integrated Discovery (DAVID). Results: We developed a 49gene signature with SOD2- and NRF2-associated genes. Using $m R N A$ expression data for the 49-gene signature, we performed hierarchical clustering to stratify patients into two subtypes, subtype A and B. In the TCGA cohort, subgroup A demonstrated a better prognosis than subgroup $B$ in patients who received $R T$. The signature robustness was evaluated in other independent cohorts. We showed through colony-
\end{abstract}

This article is freely accessible online.

Correspondence to: Young-Gyu Eun, Department of Otolaryngology-Head and Neck Surgery, Kyung Hee University Medical Center, \#1 Hoegi-dong, Dongdaemun-gu, Seoul 130702, Republic of Korea. Tel: +82 29588474, Fax: +82 29588470, e-mail: ygeun@khu.ac.kr

Key Words: SOD2, NRF2, prognosis predictor, radioresistance, head and neck cancer. formation assay that depletion of SOD2 or NRF2 leads to increased radiosensitivity. Conclusion: We identified and validated a robust gene signature of SOD2- and NRF2associated genes in HNSCC and confirmed their link to radioresistance using in vitro assay, providing a novel biomarker for the evaluation of HNSCC prognosis.

Head and neck squamous cell carcinoma (HNSCC) was the seventh most common cancer in 2018 (8.5\% new cases and $4.8 \%$ deaths) (1). Smoking, alcohol consumption, and human papillomavirus (HPV) infection are well-known high-risk factors for HNSCC carcinogenesis (2-4). Typically, HNSCC treatment includes radiation therapy (RT), surgery, and chemotherapy. Especially in treatment of HNSCC, RT is as important as surgical therapy. Recently, in an effort to reduce radiation toxicity and improve its efficacy, clinicians have begun to treat patients with combination chemoradiotherapies (5). Despite improvement in treatment and diagnosis, approximately $50 \%$ of patients with HNSCC experience local recurrence and metastasis (6). However, recent advances in molecular biology have improved the understanding of the molecular mechanisms of this disease. These advances may help to identify patients who are more likely to respond RT (7).

RT is widely used in cancer treatments but is known to have severe side-effects. This is because radiation directly damages DNA and generates free radicals in cells, which indirectly damages DNA. This DNA damage induces death of cancer cells, resulting in radiotherapeutic success. However, cellular antioxidant responses may reduce the efficacy of radiationinduced DNA damage (8). Reactive oxygen species (ROS) cause DNA damage, but they can be neutralized by superoxide dismutase 2 (SOD2), an endogenous antioxidant enzyme, 
protecting cells from ROS-associated DNA damage (9). SOD2 expression is often used as the primary marker for resistance the effects of RT, a phenomenon that can lead to RT failures (10).

Nuclear factor erythroid-2-related factor 2 (NRF2) is a well-known regulator of oxidative stress-related cellular defense mechanisms (11). Gain-of-function NRF2 mutations or $N R F 2$ activation in cancer cells involved in oxidative stress resistance in response to RT or chemotherapy have been observed in various cancer types, including non-smallcell lung cancer and prostate cancer (12). Previous studies have suggested that SOD2 and NRF2 are closely related to radioresistance. Thus, we sought to investigate whether SOD2- and NRF2-associated gene signatures might be used as prognostic biomarkers in HNSCC when developing personalized therapeutic strategies.

\section{Materials and Methods}

Patients and cohorts. Gene expression and clinical data were obtained from public databases. Data from The Cancer Genome Atlas (TCGA) cohort were downloaded from the UCSC Cancer Genomics Browser (https://genome-cancer.ucsc.edu/). Data from the Institute for Medical Informatics, Epidemiology, and Statistics [Seattle cohort (GSE41613) (13) and Leipzig cohort (GSE65858) (14)] were acquired from the National Center for Biotechnology Information Gene Expression Omnibus database (http://www.ncbi.nlm.nih.gov/geo). Table I summarizes the clinical and pathological characteristics of the patients of each cohort. These data from TCGA, Leipzig and Seattle cohort ( $n=291,189$ and 53, respectively) include clinical information of patients who received RT. Because this study relied on publicly available datasets no Institutional Review Board approvals were necessary.

Development of gene signature. Pearson correlation analysis was used to evaluate genes which may be related to the gene expression of SOD2 or NRF2. The correlation coefficient (r) was used to identify genes with a high degree of correlation with SOD2 and NRF2 expression. When correlation analysis was performed between SOD2 and other genes, genes with Pearson correlation coefficients $>0.4$ and corresponding $p<0.01$ were considered as genes with positive correlation with SOD2 expression. In the case of NRF2, genes with $\mathrm{r}>0.6$ and $p<0.01$ were considered as genes having a positive correlation with NRF2. The mRNA expression data from the TCGA cohort were used as a training set to develop and evaluate the prognostic value of this gene signature and then the Leipzig and Seattle cohorts were used to validate this signature. The gene list we used was generated by Illumina HiSeq instrument (labeled as illuminahiseq_rnaseqv2-RSEM_genes_normalized). Genes were mapped onto the human genome coordinates using UCSC Xena HUGO probeMap [hg19, GRCh37 Genome Reference Consortium Human Reference 37 (GCA_000001405.1)]. Table II summarizes the initial list SOD2- and NRF2-associated genes and includes the correlation coefficient for each. Patients were classified into subgroups with similar gene signatures by hierarchical clustering using Pearson correlation and average linkage methods. Cluster analysis was performed using Gene Cluster 3.0 and visualized by Treeview (15).
Pathway analysis. The Kyoto Encyclopedia of Genes and Genomes (KEGG) is a database containing various biological pathways and genomes (16). The Database for Annotation, Visualization and Integrated Discovery (DAVID, http://david.abcc.ncifcrf.gov/) is an online bioinformatic tools used for functional annotation, gene functional classification and gene ID conversion $(16,17)$. We performed pathway analysis using DAVID and reported our results using the KEGG pathway database $(p<0.05)$.

Validation of gene signature by prediction model in other independent cohorts. We validated the gene signature as prognostic predictor using data for patients with HNSCC from independent cohorts and the Support Vector Machine (SVM) (18). The SVM predictor is a linear function of log-ratios or log-intensities that allows better data separation based on the penalty cost of any misclassified specimen number. Gene-expression data from TCGA (training set) was grafted into the SVM algorithm to generate discriminators and then these were used to validate the gene signatures in the Seattle and Leipzig cohorts. Predictive model construction and validation were performed using BRB-array tools (https://brb.nci.nih.gov/BRB-ArrayTools/).

Cell culture. The human HNSCC cell line CAL27 (tongue carcinoma cells) was used and purchased from the American Type Culture Collection (ATCC, Manassas, VA, USA). Cells were cultured in a Dulbecco's modified Eagle's medium containing 10\% heat-inactivated fetal bovine serum (Hyclone, Logan, UT, USA) and $1 \%$ penicillin-streptomycin (Corning Inc., Corning, NY, USA) in humidified incubators at $37^{\circ} \mathrm{C}$ with $5 \% \mathrm{CO}_{2}$.

Transfection. Cells were transfected with negative control siRNA, siSOD2, and siNRF2 using Lipofectamine RNAiMAX Transfection Reagent (Thermo Fisher Scientific, Waltham, MA, USA) according to the manufacturer's instructions. The negative control siRNA products were purchased from Bioneer (Daejeon, Republic of Kore), while the siSOD2 constructs were purchased from Santa Cruz Biotechnology (Dallas, TX, USA) and the siNRF2 products were bought from IDT (Coralville, IA, USA).

Western blot. Lysates from siRNA-treated CAL27 cells were homogenized in RIPA buffer [1\% Triton X-100, $1 \%$ sodium deoxycholate, $0.1 \%$ sodium dodecyl sulfate (SDS), $150 \mathrm{mM} \mathrm{NaCl}$, $50 \mathrm{mM}$ Tris- $\mathrm{HCl}(\mathrm{pH} 7.5)$, and $2 \mathrm{mM}$ ethylenediamine tetra-acetic acid (pH8.0)] purchased from Biosesang (Seongnam-si, Republic of Korea). Protein concentration was then quantified using a Pierce Micro BCA Protein Assay Kit (Thermo Fisher Scientific), according to the manufacturer's protocol and equal amounts of protein, mixed with loading dye (5X SDS-polyacrylamide gel electrophoresis loading buffer; iNtRON Biotechnology, Seongnamsi, Republic of Korea), were added to each lane and resolved using an SDS-polyacrylamide gel. Following electrophoresis, proteins were transferred to polyvinylidene difluoride membranes (Millipore, Burlington, MA, USA) and blocked for $1 \mathrm{~h}$ in 5\% skim milk and Tris-buffered saline with $0.1 \%$ Tween-20. These membranes were then treated with appropriate primary antibodies and incubated overnight at $4{ }^{\circ} \mathrm{C}$. The following antibodies were used: anti-SOD2 (ADI-SOD-110, rabbit monoclonal antibody; Enzo Life Science, Farmingdale, NY, USA); anti-NRF2 (ab62352, rabbit polyclonal antibody; Abcam, Cambridge, UK); anti-actin (sc47778, mouse monoclonal antibody; Santa Cruz Biotechnology). 
Noh et al: Gene Signature Predicting Radioresistance in HNSCC

Table I. Patient frequency data from independent cohorts. All cohorts were patients with head and neck cancer treated with radiation therapy.

\begin{tabular}{|c|c|c|c|c|}
\hline & & TCGA (n=291) & Leipzig $(n=189)$ & Seattle $(n=53)$ \\
\hline \multirow[t]{2}{*}{ Gender } & Male & $229(78.7 \%)$ & $151(79.9 \%)$ & $35(66.0 \%)$ \\
\hline & Female & $62(21.3 \%)$ & $38(20.1 \%)$ & $18(34.0 \%)$ \\
\hline \multirow[t]{2}{*}{ Age } & $\geq 60$ Years & $144(49.5 \%)$ & $76(40.2 \%)$ & $22(41.5 \%)$ \\
\hline & $<60$ Years & $147(50.5 \%)$ & $113(59.8 \%)$ & $31(58.5 \%)$ \\
\hline \multirow[t]{4}{*}{ Anatomic site } & Oral & $162(55.7 \%)$ & $55(29.1 \%)$ & \\
\hline & Oropharynx & $53(18.2 \%)$ & $75(39.7 \%)$ & \\
\hline & Larynx & $70(24.1 \%)$ & $27(14.3 \%)$ & \\
\hline & Hypopharynx & $6(2.1 \%)$ & $29(15.3 \%)$ & \\
\hline \multirow[t]{4}{*}{ Primary tumor } & $\mathrm{T} 1$ & $21(7.2 \%)$ & $16(8.5 \%)$ & \\
\hline & $\mathrm{T} 2$ & $57(19.6 \%)$ & $50(26.5 \%)$ & \\
\hline & $\mathrm{T} 3$ & $57(19.6 \%)$ & $50(26.5 \%)$ & \\
\hline & $\mathrm{T} 4$ & $111(38.1 \%)$ & $73(38.6 \%)$ & \\
\hline \multirow[t]{4}{*}{ Regional lymph node status } & NO & $73(25.1 \%)$ & $50(26.5 \%)$ & \\
\hline & $\mathrm{N} 1$ & $40(13.7 \%)$ & $25(13.2 \%)$ & \\
\hline & $\mathrm{N} 2$ & $122(41.9 \%)$ & $104(55.0 \%)$ & \\
\hline & N3 & $2(0.7 \%)$ & $10(5.3 \%)$ & \\
\hline \multirow[t]{4}{*}{ Stage } & I & $8(2.7 \%)$ & $4(2.1 \%)$ & I/ II: $8(15.1 \%)$ \\
\hline & II & $16(5.5 \%)$ & $14(7.4 \%)$ & \\
\hline & III & $38(13.1 \%)$ & $31(16.4 \%)$ & III/ IV: $45(84.9 \%)$ \\
\hline & IV & $180(61.9 \%)$ & $140(74.1 \%)$ & \\
\hline \multirow[t]{2}{*}{ HPV status } & Positive & $43(14.8 \%)$ & $54(28.6 \%)$ & \\
\hline & Negative & $248(85.2 \%)$ & $135(71.4 \%)$ & $53(100 \%)$ \\
\hline \multirow[t]{2}{*}{ Smoking } & Yes & $219(75.3 \%)$ & $155(82.0 \%)$ & \\
\hline & No & $68(23.4 \%)$ & $34(18.0 \%)$ & \\
\hline \multirow[t]{2}{*}{ Alcohol } & Yes & $210(72.2 \%)$ & $166(87.8 \%)$ & \\
\hline & No & $78(26.8 \%)$ & $23(12.2 \%)$ & \\
\hline
\end{tabular}

HPV: Human papillomavirus.

The blots were then washed and treated with secondary antibody for $1 \mathrm{~h}$ at room temperature, and the protein-antibody complexes were detected using enhanced chemiluminescence (RPN2232; GE Healthcare, Chicago, IL, USA) according to the manufacturer's protocol.

Colony formation assay and RT. siRNA-transfected cells were first trypsinized, diluted, and enumerated; then, they were and seeded onto 6-well plates in triplicate. After $24 \mathrm{~h}$ of incubation, the cells were exposed to different doses of $\mathrm{X}$-ray radiation $(0,2,4$, and $8 \mathrm{~Gy})$ using an XStrahl RS225 cabinet at room temperature with $195 \mathrm{kV} / 15 \mathrm{~mA}$ $\mathrm{X}$-rays, producing a dose rate of $1.6 \mathrm{~Gy}$ per minute. Irradiated and untreated control cells were subsequently cultured for 8-14 days. The surviving cell-derived colonies were stained with crystal violet solution ( $0.5 \%$ crystal violet in $50 \%$ methanol) and counted.

Statistical analysis. Only gene-expression data with matched survival data were used to determine prognostic value and prognostic significance was evaluated using the Kaplan-Meier method. Univariate and multivariate Cox proportional hazard regression analyses were performed to assess the associations between independent prognostic factors and patient survival. Correlation was determined using the Pearson correlation coefficient and clinicopathological variables were compared using the chi-square test. All statistical evaluations were completed as two-tailed $t$-tests and $p$-values less than 0.05 were considered statistically significant. All other statistical analyses were performed in the R language environment (http://www.rproject.org).

\section{Results}

Development of SOD2 and NRF2 expression-associated gene signature. Figure 1A presents an overview of our study. We used the gene-expression data from patients with HNSCC in the TCGA cohort to identify any genes closely correlated with expression of SOD2 or NRF2. This led to the production of a 49-gene signature consisting of 31 genes linked to SOD2 $(\mathrm{r}>0.4, p<0.01)$ and 18 genes linked to NRF2 $(r>0.6, p<0.01)$ expression (Table II). To determine the extent of similarity between patients, we performed unsupervised clustering of mRNA expression data for 49 genes from the TCGA cohort (Figure 1B). As the heatmap indicates, patients were divided into two cluster, subgroups $A(n=126)$ and $B(n=165)$, and their overall survival (OS) rates were compared using the Kaplan-Meier method to evaluate the prognostic significance. Subgroup A had significantly better OS than subgroup B (Figure 1C) $(p=0.003)$. 


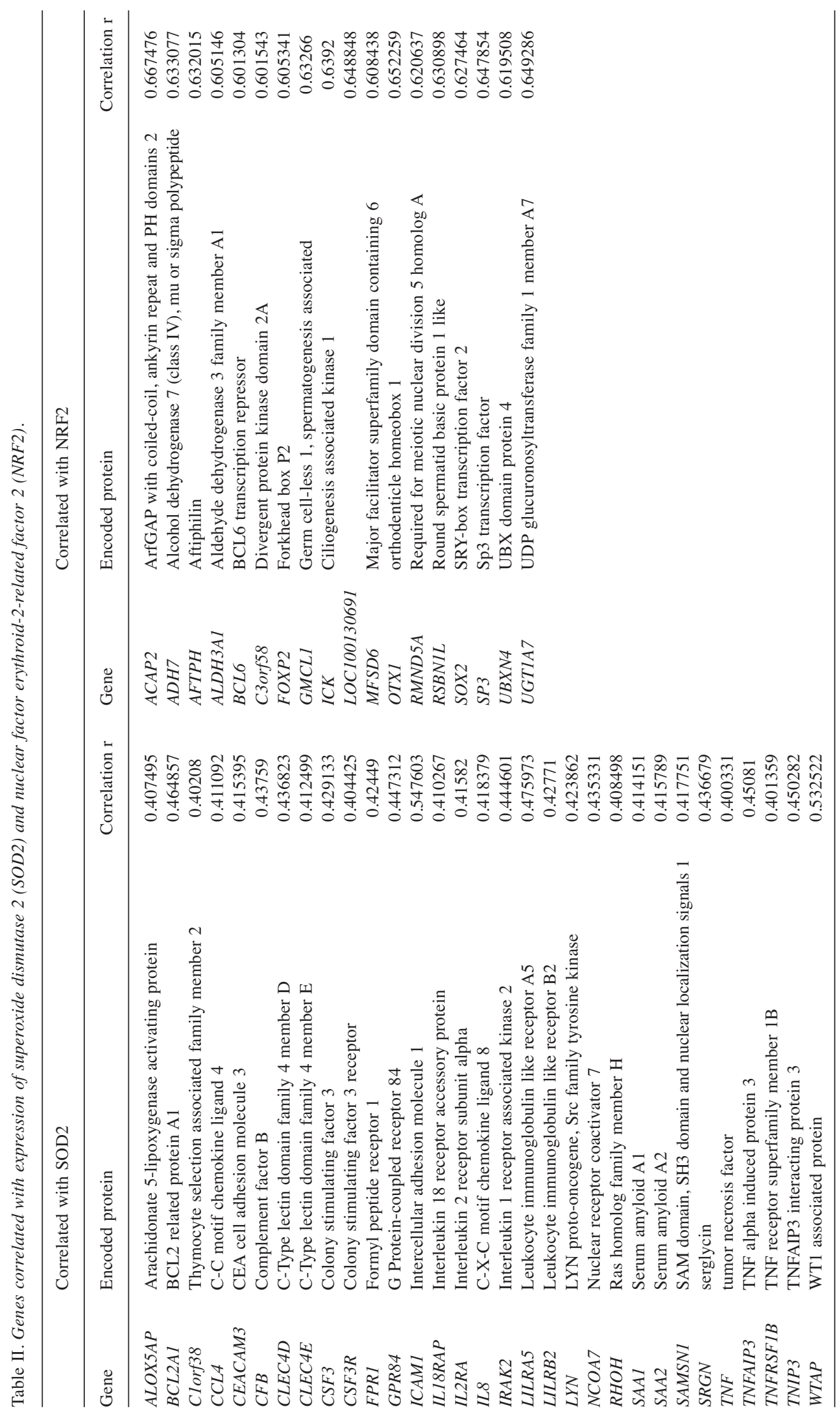




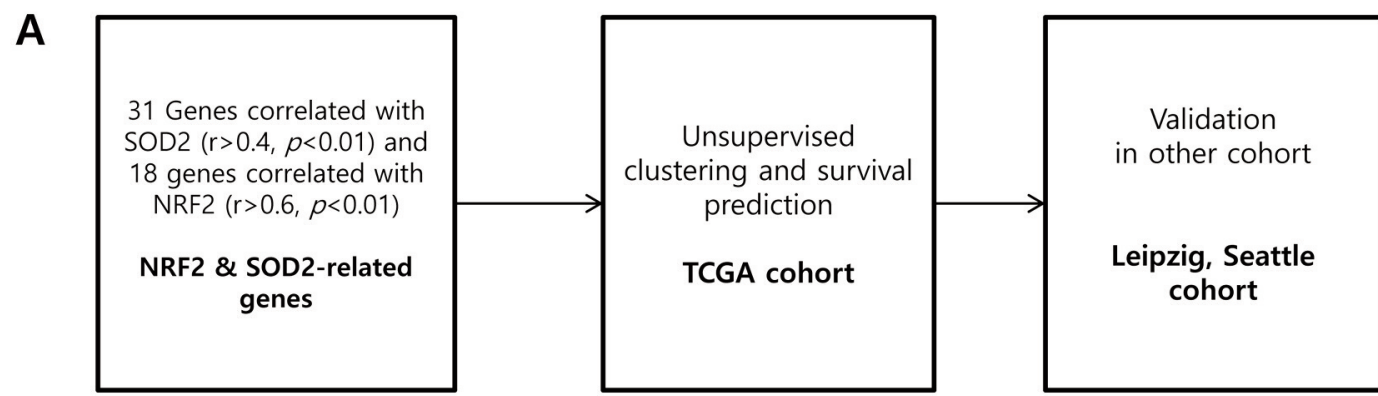

\section{B}

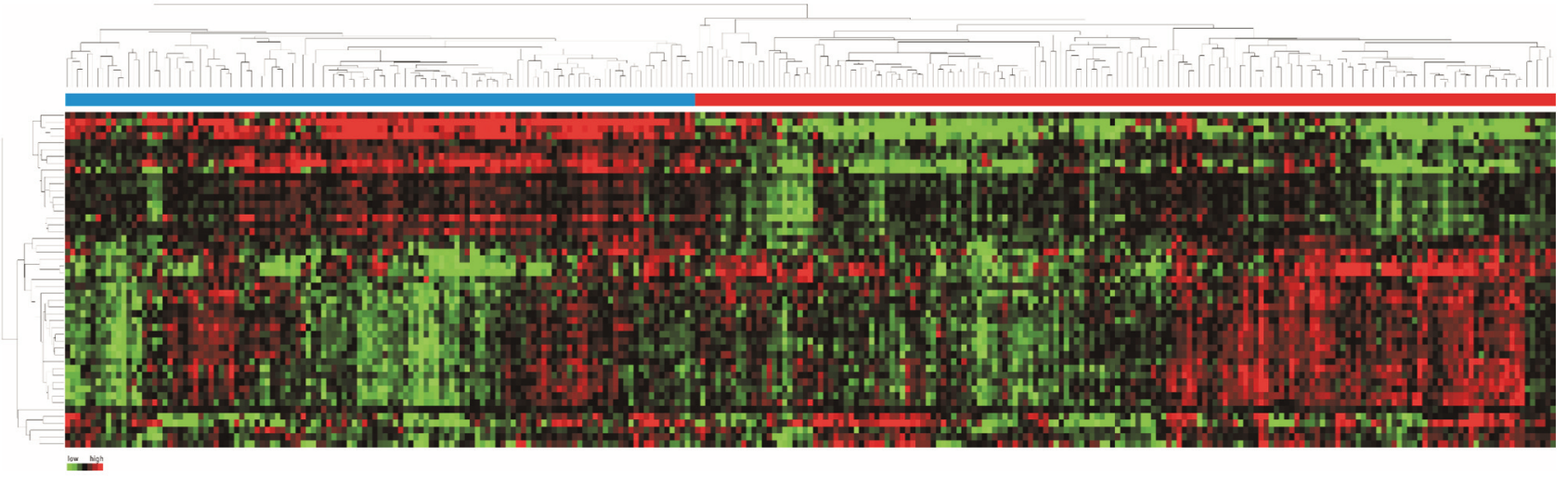

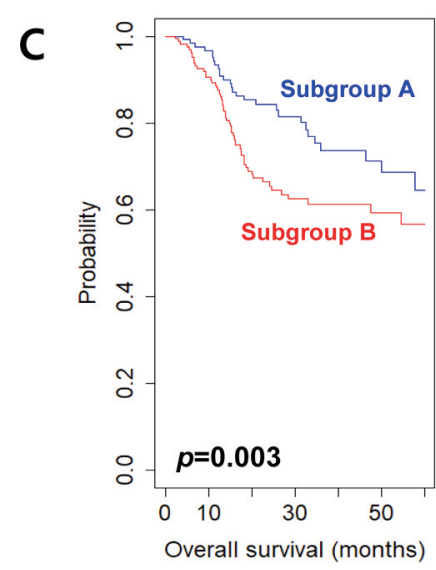

Validation of the SOD2- and NRF2-associated gene signature. The validation was performed to determine the robustness of gene signature in other independent cohorts. For validation of this gene signature, patients who received radiotherapy were selected from the Leipzig and Seattle cohorts $(n=189$, and $n=53$, respectively). Prediction was performed through an SVM algorithm using BRB-array tools. Accordingly, the Leipzig cohort patients were divided into subgroup A $(n=80)$ and subgroup B patients $(n=109)$, and the Seattle cohort patients were divided into subgroup A
Figure 1. Stratification of patients with head and neck squamous cell carcinoma from The Cancer Genome Atlas (TCGA) using a 49-gene signature. A: Schematic overview of study. B: Patients were clustered by hierarchical clustering using Pearson's correlation-coefficient and average-linkage method. C: Kaplan-Meier plot for overall survival comparison between the two groups according to the gene signature.

$(n=24)$ and subgroup B patients $(n=29)$. Patients of subgroup A had a better prognosis than those of subgroup B for both these cohorts ( $p=0.04$, and $p=0.004$, respectively; Figure 2 ). Therefore, we verified the superiority of our SOD2- and NRF2-associated gene signature for clinical usefulness as a predictor of the prognosis of patients receiving $\mathrm{RT}$.

SOD2- and NRF2-associated gene signature as prognostic predictor. Univariate and multivariate Cox proportional hazard regression analyses were used to evaluate the effect of the 

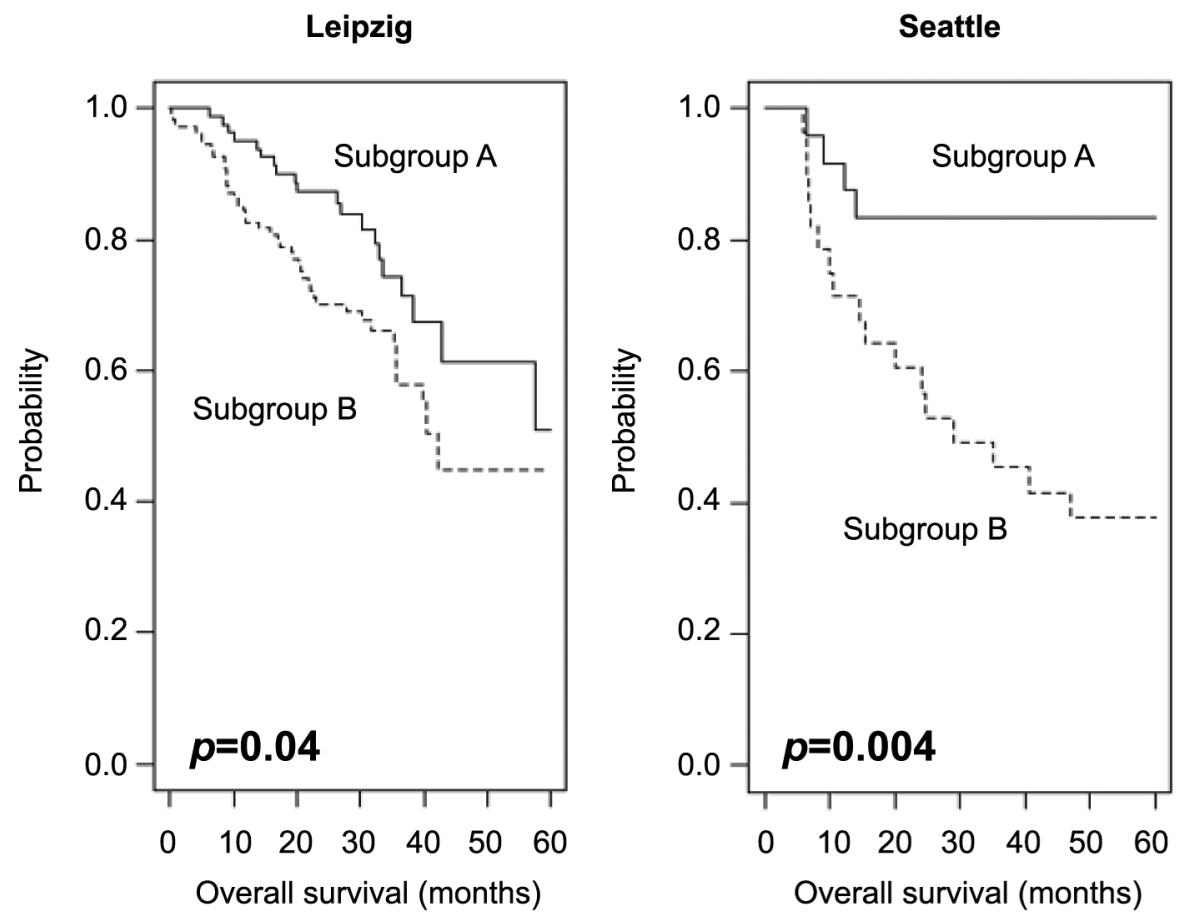

Figure 2. Construction of the predictive model and evaluation of predicted outcome. Kaplan-Meier plots of Leipzig and Seattle cohorts. The differences between the groups were statistically significant, as indicated by the log-rank test.

SOD2- and NRF2-associated gene signature on survival in the TCGA and Leipzig cohorts $(n=480)$, using the available clinical data. The combination of the SOD2- and NRF2-associated gene signature and other clinicopathological factors (e.g. patient age, gender, smoking, alcohol consumption, anatomical site, HPV infection, and American Joint Committee on Cancer TNM classification) were used for Cox proportional hazard regression analysis. In the univariate analysis, the SOD2- and NRF2associated gene signature (subgroup A vs. B), anatomical site (oropharynx vs. other), HPV infection (HPV-positive vs. HPVnegative) were shown to be closely associated with OS. In the multivariate analysis, the subgroup B SOD2- and NRF2associated gene signature and HPV-positivity emerged as significant factors for poor prognosis [hazard ratio $1.58(95 \%$ confidence interval $=1.07-2.35), p=0.0225$; hazard ratio $=0.41$ (95\% confidence intervaI $=0.23-0.73), p=0.00242$, respectively] (Table III). Therefore, this signature appears to be an independent prognosis factor in HNSCC.

Association between the SOD2- and NRF2-associated gene signature and HNSCC characteristics. We compared the known prognostic clinicopathological indicators in subgroups A and B (Table IV). Chi-square tests were used to evaluate the clinical data from patients in the TCGA and Leipzig cohorts $(n=480)$ and showed that there were no statistically significant differences in $\mathrm{T}$ classification, $\mathrm{N}$ classification, American Joint Committee on Cancer stage and alcohol consumption $(p>0.05)$ between these cohorts (Table IV). Subgroup A was shown to include a lower proportion of HPV-negative patients [72.3\% (149/206) vs. $83.9 \%(230 / 274), p=0.0058]$ and a higher proportion of patients with a history of smoking [26.2\% (54/206) vs. $15.3 \%(42 / 274), p=0.0039]$.

Relationship of SOD2 and NRF2 with RT resistance. To explore the relationship between NRF2/SOD2 and RT resistance, we performed a colony-formation assay using NRF2 or SOD2-depleted HNSCC cells after ionizing radiation. CAL27 cells were transfected with specific $N R F 2$ or SOD2 siRNAs and their knock-down was confirmed via western blot (Figure 3A). Then siCON, siNRF2 or siSOD2transfected cells were exposed to different doses of radiation and monitored for colony-forming ability. Our results showed that siNRF2 and siSOD2 significantly reduced the survival fraction of CAL27 cells following RT when compared with siCON (Figure 3B). This result confirms that NRF2 and SOD2 are closely related to RT resistance and are likely to be potential predictors for increased RT resistance.

KEGG pathway analysis of SOD2- and NRF2-associated gene signature. Pathway analysis was applied to predict the biological functions of the SOD2 and NRF2-associaeted gene 
Table III. Univariate and multivariate Cox proportional hazard regression analysis of overall survival in The Cancer Genome Atlas and Leipzig $\operatorname{cohort}(n=480)$.

\begin{tabular}{|c|c|c|c|c|c|}
\hline \multirow[b]{2}{*}{ Variable } & & \multicolumn{2}{|c|}{ Univariate } & \multicolumn{2}{|c|}{ Multivariate } \\
\hline & & HR $(95 \%$ CI) & $p$-Value & HR $(95 \%$ CI) & $p$-Value \\
\hline 49-Gene signature & Subgroup B & $1.75(1.24-2.47)$ & 0.00148 & $1.58(1.07-2.35)$ & 0.0225 \\
\hline Gender & Male & $0.78(0.53-1.13)$ & 0.186 & $0.73(0.48-1.10)$ & 0.1334 \\
\hline Age & $>60$ Years & $1.15(0.84-1.59)$ & 0.383 & $1.15(0.81-1.62)$ & 0.4341 \\
\hline Smoking & Yes & $1.10(0.73-1.64)$ & 0.656 & $1.03(0.67-1.58)$ & 0.8827 \\
\hline Alcohol & Yes & $1.23(0.82-1.91)$ & 0.292 & $1.37(0.86-2.18)$ & 0.1825 \\
\hline Anatomic site & Oropharynx & $0.48(0.26-0.86)$ & 0.0140 & $0.71(0.38-1.33)$ & 0.2888 \\
\hline HPV status & Positive & $0.42(0.25-0.71)$ & 0.0011 & $0.41(0.23-0.73)$ & 0.00242 \\
\hline Stage & III \& IV & $1.30(0.72-2.35)$ & 0.385 & $1.31(0.72-2.38)$ & 0.3805 \\
\hline
\end{tabular}

CI: Confidence interval; HPV: human papillomavirus; HR: hazard ratio. Statistically significant $p$-values are shown in bold.

Table IV. Clinicopathological characteristics of patients divided into subgroup A and subgroup B by the 49-gene signature.

\begin{tabular}{|c|c|c|c|c|}
\hline & & Subgroup A $(n=206)$ & Subgroup B $(n=274)$ & $p$-Value \\
\hline \multirow[t]{2}{*}{ T Classification, n (\%) } & I, II & $64(31.1 \%)$ & $74(27.0 \%)$ & \multirow[t]{2}{*}{0.3774} \\
\hline & III, IV & $138(67.0 \%)$ & $195(71.2 \%)$ & \\
\hline \multirow[t]{2}{*}{$\mathrm{N}$ Classification, $\mathrm{n}(\%)$} & Positive & $129(62.6 \%)$ & $182(66.4 \%)$ & \multirow[t]{2}{*}{0.4643} \\
\hline & Negative & $71(34.5 \%)$ & $85(31.0 \%)$ & \\
\hline \multirow[t]{2}{*}{ Stage, n (\%) } & I, II & $21(10.2 \%)$ & $27(9.9 \%)$ & \multirow[t]{2}{*}{$>0.99$} \\
\hline & III, IV & $182(88.3 \%)$ & $243(88.7 \%)$ & \\
\hline \multirow{2}{*}{ HPV status, n (\%) } & Positive & $54(26.2 \%)$ & $42(15.3 \%)$ & \multirow{2}{*}{0.003967} \\
\hline & Negative & $149(72.3 \%)$ & $230(83.9 \%)$ & \\
\hline \multirow[t]{2}{*}{ Smoking, n (\%) } & Yes & $173(84.0 \%)$ & $201(73.4 \%)$ & \multirow[t]{2}{*}{0.005833} \\
\hline & No & $31(15.0 \%)$ & $71(25.9 \%)$ & \\
\hline \multirow[t]{2}{*}{ Alcohol, n (\%) } & Yes & $162(78.6 \%)$ & $214(78.1 \%)$ & \multirow[t]{2}{*}{0.7367} \\
\hline & No & $41(19.9 \%)$ & $60(21.9 \%)$ & \\
\hline
\end{tabular}

Statistically significant $p$-values are shown in bold.

signature based on KEGG databases. All 49 SOD2- and NRF2associated genes were analyzed using DAVID. The KEGG pathway results indicated that the SOD2- and NRF2-associated genes were mainly involved in the nuclear factor kappa-lightchain-enhancer of activated B cells (NF-kB) signaling pathway, cytokine-cytokine receptor interaction, hematopoietic cell lineage, tumor necrosis factor (TNF) signaling pathways and malaria. These results are summarized in Table V.

\section{Discussion}

Despite numerous therapeutic developments, such as RT and adjuvant chemotherapy, patients with HNSCC experience cancer recurrence and metastasis. The necessity of individual characteristic-based patient classification for prediction of therapeutic prognosis has already been suggested in previous study (19). In this study, SOD2- and NRF2-associated gene signature was identified and shown to be effective in classification of patients with HNSCC. When subgrouping HNSCC from TCGA through unsupervised clustering into two subgroups, patients in subgroup A had a better OS than those of subgroup B. We validated that the prognostic significance between the two subgroups was also significant in other independent cohorts.

HNSCC classification was based on genetic alterations or gene-expression profiles. This classification reflects the overall biological characteristics of these patients, some of which can be used to generate hypotheses about patient selection and new treatment targeting (20). Therefore, patient stratification and the identification of prognostic biomarkers are of increasing importance in the development of personalized medicines, as well as in applications including diagnostic selection, prognosis, and targeted therapies. To explore the effective stratification of patients for predicting 
A
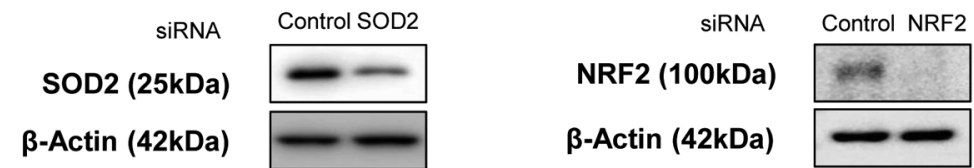

B
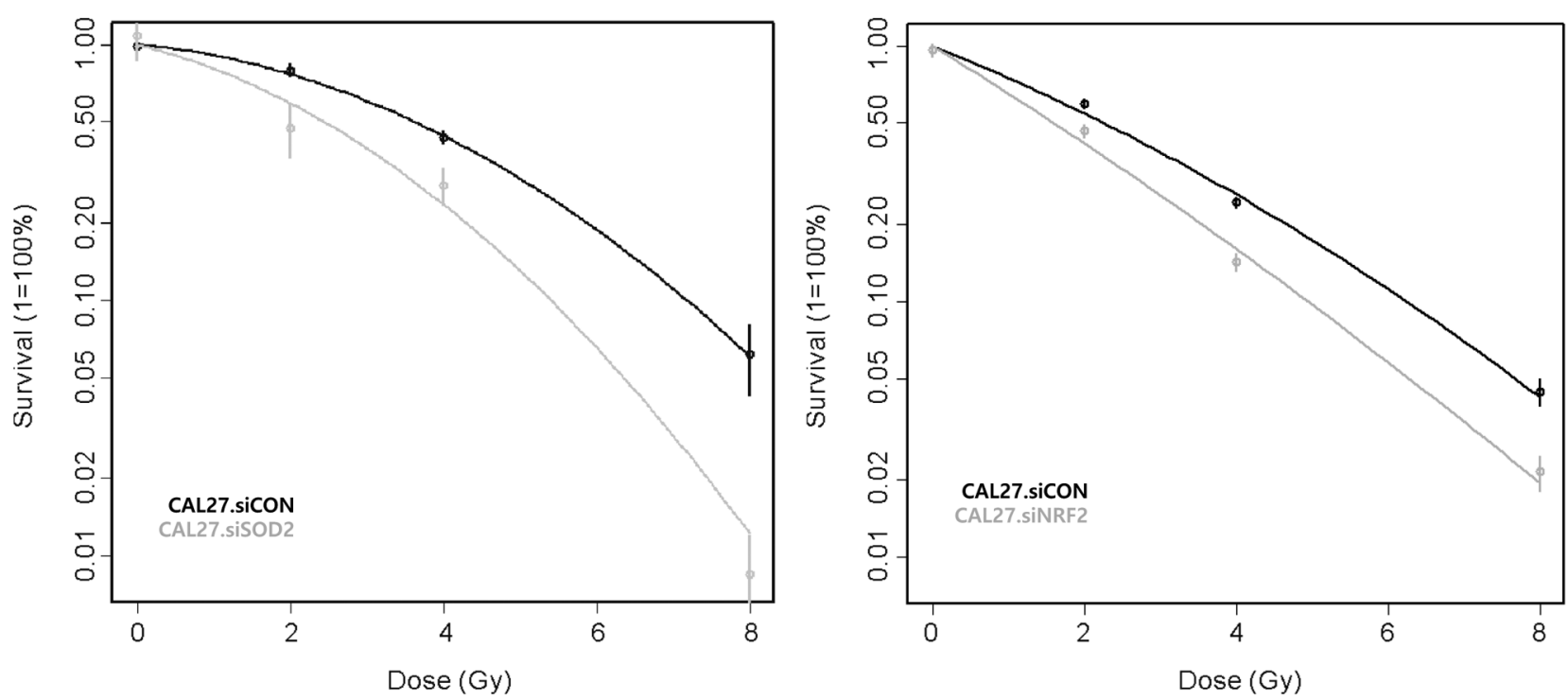

Figure 3. Knock-down of superoxide dismutase 2 (SOD2) or nuclear factor erythroid-2-related factor 2 (NRF2) sensitized head and neck squamous cell carcinoma cells to radiation. A: SOD2 and NRF2 were effectively silenced using siRNA. B: Colony formation assay was performed, and radiosensitivity was increased by knock-down of SOD2 or NRF2 ( $p=0.003$ and $p=0.002$, respectively).

prognosis, we intended to develop a gene signature using SOD2 and NRF2, which are already known to correlate with increased cancer metastasis and RT resistance.

SOD2 is involved in tumor progression and invasion via several mechanisms. SOD2 is induced by up-regulation of Bcell lymphoma-extra large (BCLx-xL), intracellular carcinogenic genes, or extracellular factors such as surfeit 2 (SURF2) and interleukin-6 (IL6), leading to cancer cell invasion (21). Additionally, SOD2 modulates the cellular redox environment driving malignancy through several outcomes including prolonged tumor cell survival and increased invasion and migration (22). Hosoki et al. revealed that SOD2 overexpression enhances cellular resistance to $\gamma$ irradiation. Using a microarray and pathway analysis, SOD2 has also been shown to function as a signaling mediator involved in the cellular resistance against ROS-induced cytotoxicity (23). We showed that SOD2 inhibition reduced the colony-forming ability of cells. This indicates the potential role of SOD2 in cell protection from irradiation. Based on the results of other previous studies and our current findings, SOD2 clearly protects against cellular damage by radiation and induces cell resistance to ionizing radiation (21-24).
Table V. Pathways significantly related to superoxide dismutase 2 and nuclear factor erythroid-2-related factor 2-associated genes.

\begin{tabular}{lcc}
\hline Term & Count & $p$-Value \\
\hline Nuclear factor-kappa B signaling pathway & 6 & 0.00001 \\
Cytokine-cytokine receptor interaction & 7 & 0.00014 \\
Hematopoietic cell lineage & 4 & 0.00330 \\
Tumor necrosis factor signaling pathway & 4 & 0.00580 \\
Malaria & 3 & 0.01200 \\
Staphylococcus aureus infection & 3 & 0.01500 \\
Drug metabolism - cytochrome P450 & 3 & 0.02300 \\
Metabolism of xenobiotics by cytochrome P450 & 3 & 0.02700 \\
Chemical carcinogenesis & 3 & 0.03100 \\
\hline
\end{tabular}

Previous studies have shown that changes in NRF2 expression are associated with poor prognosis as it can result in local recurrence, metastasis, and radioresistance in patients. NRF2 forms a positive feedback loop with p62 and contributes to mesenchymal metastasis, proliferation, and invasion in glioma cells (25). NRF2 increases resistance by increasing carbonyl reductase-1 (CBR1) transcription after RT in HNSCC (26). The suppression of NRF2 and the NRF2 signaling 
pathway strengthens radiosensitivity of hepatoma cells (27). NRF2 activation in lung squamous cell carcinoma led to a subsequent reduction of intracellular ROS and reduced the kelch-like ECH-associated protein1 (KEAP1) level, resulting in increased radiation resistance, tumor proliferation, and metastasis (28). We reported that NRF2 is a metastasis- or radiation resistance-related factor in cancer, with one recent study identifying its function in tumor progression (25-28).

Pathway analysis of the SOD2- and NRF2-associated gene signature was performed. As a result, association with the NF$\mathrm{kB}$ signaling pathway, cytokine-cytokine receptor interaction, hematopoietic cell lineage, TNF signaling pathway and malaria were revealed. NF- $\mathrm{KB}$ is a nuclear transcription factor that acts downstream of Aurora-A and has been implicated in increasing resistance of lung adenocarcinoma to RT (29). In addition, NF-kB up-regulates TNF $\alpha$ creating a self-sustaining positive feedback loop (30). This positive feedback loop between $\mathrm{NF}-\mathrm{KB}$ and $\mathrm{TNF} \alpha$ can result in the induction of radiation resistance. Therefore, self-sustaining $\mathrm{NF}-\mathrm{kB}$ expression determines radiation resistance in tumor cells. Our findings that the SOD2- and NRF2-associated gene signature can be used as a prognostic tool to evaluate resistance of HNSCC to RT supports the previous findings.

HNSCC progression is associated with several high-risk factors including HPV infection, tobacco usage and alcohol consumption. However, unlike breast cancer type 1 susceptibility protein (BRCA1) and BRCA2 in breast cancer (31), and Kirsten rat sarcoma viral oncogene homolog (KRAS), TP53, SMAD family member 4 (SMAD4), and vraf murine sarcoma viral oncogene homolog $\mathrm{B}$ (BRAF) in colorectal cancer (32), HNSCC does not have any known driver genes associated with its initiation. With the release of the TCGA consortium in 2015, a significant amount of cancer-related molecular data became accessible. Using these data, further genomic profiling-based patient subclassification is expected to improve our understanding of HNSCC.

Our results also revealed that inhibition of SOD2 reduces RT resistance in HNSCC. This supports the results of our previous study, which demonstrated that NRF2 increases such resistance by regulating $C B R I$ at the transcriptional level (26).

In this study, we proposed that a gene signature using two factors previously associated with RT resistance or invasion (SOD2 and NRF2) have the potential to predict patient prognosis. To overcome the limitations to clinical utility of using a 49-gene signature, we tried several methods to reduce the number of genes. Despite several trials, this subset of 49 genes showed the best performance. The expression of a single gene cannot represent a complex biological pathway, and although the role of these individual genes may be well known, it is difficult to use single genes to predict clinical and therapeutic outcomes. Thus, identifying a set of genes that are closely correlated with these key regulators and creating a gene signature may be an effective way to overcome this limitation.

\section{Conclusion}

We developed a gene signature that can predict resistance to RT using an SOD2- and NRF2-associated gene signature. This gene signature was validated in independent HNSCC cohorts and shown to be a valuable predictor for RT resistance.

\section{Conflicts of Interest}

The Authors declare no potential conflicts of interest with respect to the research, authorship, and publication.

\section{Authors' Contributions}

Study design: J.K.N., S.R.W., M.Y.Y., Y.G.E. and S.G.K. Drafting and editing the article: J.K.N., S.R.W. and Y.G.E. Data analysis: J.K.N., S.I.K. and Y.C.L. Biomolecular experiments: J.K.N., and M.K.L. Processing RT experiment: M.K.K., and S.K.M. Supervising the project: Y.G.E. and S.G.K.

\section{Acknowledgements}

This work was supported by the National Research Foundation of Korea (NRF) grant funded by the Korea government (MSIT) (no. 2020R1A5A2019413) and by a grant of the Korea Health Technology R\&D Project through the Korea Health Industry Development Institute (KHIDI), funded by the Ministry of Health \& Welfare, Republic of Korea (grant number: HI20C1205).

\section{References}

1 Bray F, Ferlay J, Soerjomataram I, Siegel RL, Torre LA and Jemal A: Global cancer statistics 2018: GLOBOCAN estimates of incidence and mortality worldwide for 36 cancers in 185 countries. CA Cancer J Clin 68(6): 394-424, 2018. PMID: 30207593. DOI: $10.3322 /$ caac. 21492

2 Gillison ML, Chaturvedi AK, Anderson WF and Fakhry C: Epidemiology of human papillomavirus-positive head and neck squamous cell carcinoma. J Clin Oncol 33(29): 3235-3242, 2015. PMID: 26351338. DOI: 10.1200/JCO.2015.61.6995

3 Jethwa AR and Khariwala SS: Tobacco-related carcinogenesis in head and neck cancer. Cancer Metastasis Rev 36(3): 411-423, 2017. PMID: 28801840. DOI: 10.1007/s10555-017-9689-6

4 Kawakita D and Matsuo K: Alcohol and head and neck cancer. Cancer Metastasis Rev 36(3): 425-434, 2017. PMID: 28815324. DOI: $10.1007 / \mathrm{s} 10555-017-9690-0$

5 Chow LQM: Head and neck cancer. N Engl J Med 382(1): 6072, 2020. PMID: 31893516. DOI: 10.1056/NEJMra1715715

6 Alterio D, Marvaso G, Ferrari A, Volpe S, Orecchia R and Jereczek-Fossa BA: Modern radiotherapy for head and neck cancer. Semin Oncol 46(3): 233-245, 2019. PMID: 31378376. DOI: 10.1053/j.seminoncol.2019.07.002

7 de Roest RH, Mes SW, Poell JB, Brink A, van de Wiel MA, Bloemena E, Thai E, Poli T, Leemans CR and Brakenhoff RH: Molecular characterization of locally relapsed head and neck cancer after concomitant chemoradiotherapy. Clin Cancer Res 25(23): 7256-7265, 2019. PMID: 31439582. DOI: 10.1158/10780432.CCR-19-0628 
8 Srinivas US, Tan BWQ, Vellayappan BA and Jeyasekharan AD ROS and the DNA damage response in cancer. Redox Biol 25: 101084, 2019. PMID: 30612957. DOI: 10.1016/j.redox. 2018.101084

9 Kim YS, Gupta Vallur P, Phaëton R, Mythreye K and Hempel $\mathrm{N}$ : Insights into the Dichotomous Regulation of SOD2 in Cancer. Antioxidants (Basel) 6(4): 86, 2017. PMID: 29099803. DOI: 10.3390/antiox6040086

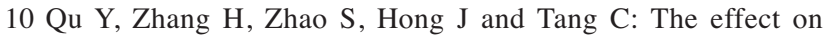
radioresistance of manganese superoxide dismutase in nasopharyngeal carcinoma. Oncol Rep 23(4): 1005-1011, 2010. PMID: 20204285. DOI: 10.3892/or_00000726

11 Zimta AA, Cenariu D, Irimie A, Magdo L, Nabavi SM, Atanasov AG and Berindan-Neagoe I: The role of Nrf2 activity in cancer development and progression. Cancers (Basel) 11(11): 1755, 2019. PMID: 31717324. DOI: 10.3390/cancers11111755

12 Zhou S, Ye W, Shao Q, Zhang M and Liang J: Nrf2 is a potential therapeutic target in radioresistance in human cancer. Crit Rev Oncol Hematol 88(3): 706-715, 2013. PMID: 24126138. DOI: 10.1016/j.critrevonc.2013.09.001

13 Lohavanichbutr P, Méndez E, Holsinger FC, Rue TC, Zhang Y, Houck J, Upton MP, Futran N, Schwartz SM, Wang P and Chen C: A 13-gene signature prognostic of HPV-negative OSCC: discovery and external validation. Clin Cancer Res 19(5): 1197-1203, 2013. PMID: 23319825. DOI: 10.1158/1078-0432.CCR-12-2647

14 Wichmann G, Rosolowski M, Krohn K, Kreuz M, Boehm A, Reiche A, Scharrer U, Halama D, Bertolini J, Bauer U, Holzinger D, Pawlita M, Hess J, Engel C, Hasenclever D, Scholz M, Ahnert P, Kirsten H, Hemprich A, Wittekind C, Herbarth O, Horn F, Dietz A, Loeffler M and Leipzig Head and Neck Group (LHNG): The role of HPV RNA transcription, immune responserelated gene expression and disruptive TP53 mutations in diagnostic and prognostic profiling of head and neck cancer. Int J Cancer 137(12): 2846-2857, 2015. PMID: 26095926. DOI: $10.1002 / \mathrm{ijc} .29649$

15 Eisen MB, Spellman PT, Brown PO and Botstein D: Cluster analysis and display of genome-wide expression patterns. Proc Natl Acad Sci USA 95(25): 14863-14868, 1998. PMID: 9843981. DOI: 10.1073/pnas.95.25.14863

16 Kanehisa M and Goto S: KEGG: kyoto encyclopedia of genes and genomes. Nucleic Acids Res 28(1): 27-30, 2000. PMID: 10592173. DOI: $10.1093 / \mathrm{nar} / 28.1 .27$

17 Huang da W, Sherman BT and Lempicki RA: Systematic and integrative analysis of large gene lists using DAVID bioinformatics resources. Nat Protoc 4(1): 44-57, 2009. PMID: 19131956. DOI: 10.1038/nprot.2008.211

18 Huang S, Cai N, Pacheco PP, Narrandes S, Wang Y and Xu W: Applications of support vector machine (SVM) learning in cancer genomics. Cancer Genomics Proteomics 15(1): 41-51, 2018. PMID: 29275361. DOI: $10.21873 / \mathrm{cgp} .20063$

19 Tonella L, Giannoccaro M, Alfieri S, Canevari S and De Cecco $\mathrm{L}$ : Gene expression signatures for head and neck cancer patient stratification: Are results ready for clinical application? Curr Treat Options Oncol 18(5): 32, 2017. PMID: 28474265. DOI: 10.1007/s11864-017-0472-2

20 Kang H, Kiess A and Chung CH: Emerging biomarkers in head and neck cancer in the era of genomics. Nat Rev Clin Oncol 12(1): 11-26, 2015. PMID: 25403939. DOI: 10.1038/nrclinonc.2014.192

21 Jung CH, Kim EM, Song JY, Park JK and Um HD: Mitochondrial superoxide dismutase 2 mediates $\gamma$-irradiation- induced cancer cell invasion. Exp Mol Med 51(2): 1-10, 2019. PMID: 30755594. DOI: 10.1038/s12276-019-0207-5

22 Hempel N, Carrico PM and Melendez JA: Manganese superoxide dismutase (Sod2) and redox-control of signaling events that drive metastasis. Anticancer Agents Med Chem 11(2): 191-201, 2011. PMID: 21434856. DOI: 10.2174/187152011795255911

23 Hosoki A, Yonekura S, Zhao QL, Wei ZL, Takasaki I, Tabuchi Y, Wang LL, Hasuike S, Nomura T, Tachibana A, Hashiguchi K, Yonei S, Kondo $\mathrm{T}$ and Zhang-Akiyama QM: Mitochondriatargeted superoxide dismutase (SOD2) regulates radiation resistance and radiation stress response in HeLa cells. J Radiat Res 53(1): 58-71, 2012. PMID: 22302046. DOI: 10.1269/jrr.11034

24 Zhang Y, Xu Z, Ding J, Tan C, Hu W, Li Y, Huang W and Xu Y: HZ08 suppresses RelB-activated MnSOD expression and enhances Radiosensitivity of prostate Cancer cells. J Exp Clin Cancer Res 37(1): 174, 2018. PMID: 30053873. DOI: 10.1186/s13046-018-0849-5

25 Pölönen P, Jawahar Deen A, Leinonen HM, Jyrkkänen HK, Kuosmanen S, Mononen M, Jain A, Tuomainen T, PasonenSeppänen S, Hartikainen JM, Mannermaa A, Nykter M, Tavi P, Johansen $T$, Heinäniemi $M$ and Levonen AL: Nrf2 and SQSTM1/p62 jointly contribute to mesenchymal transition and invasion in glioblastoma. Oncogene 38(50): 7473-7490, 2019. PMID: 31444413. DOI: 10.1038/s41388-019-0956-6

26 Yun M, Choi AJ, Lee YC, Kong M, Sung JY, Kim SS and Eun YG: Carbonyl reductase 1 is a new target to improve the effect of radiotherapy on head and neck squamous cell carcinoma. J Exp Clin Cancer Res 37(1): 264, 2018. PMID: 30376862. DOI: 10.1186/s13046-018-0942-9

27 You X, Cao X and Lin Y: Berberine enhances the radiosensitivity of hepatoma cells by Nrf2 pathway. Front Biosci (Landmark Ed) 24: 1190-1202, 2019. PMID: 31136975.

28 Jeong Y, Hoang NT, Lovejoy A, Stehr H, Newman AM, Gentles AJ, Kong W, Truong D, Martin S, Chaudhuri A, Heiser D, Zhou L, Say C, Carter JN, Hiniker SM, Loo BW Jr, West RB, Beachy P, Alizadeh AA and Diehn M: Role of KEAP1/NRF2 and TP53 Mutations in Lung Squamous Cell Carcinoma Development and Radiation Resistance. Cancer Discov 7(1): 86-101, 2017. PMID: 27663899. DOI: 10.1158/2159-8290.CD-16-0127

29 Liu JB, Hu L, Yang Z, Sun YU, Hoffman RM and Yi Z: Aurora$\mathrm{A} / \mathrm{NF}-\mathrm{kB}$ signaling is associated with radio-resistance in human lung adenocarcinoma. Anticancer Res 39(11): 5991-5998, 2019. PMID: 31704824. DOI: 10.21873/anticanres.13804

$30 \mathrm{Yu} \mathrm{H}$, Aravindan N, Xu J and Natarajan M: Inter- and intra-cellular mechanism of NF-kB-dependent survival advantage and clonal expansion of radio-resistant cancer cells. Cell Signal 31: 105-111, 2017. PMID: 28069440. DOI: 10.1016/j.cellsig.2017.01.011

31 Zhu Y, Wu J, Zhang C, Sun S, Zhang J, Liu W, Huang J and Zhang $\mathrm{Z}$ : BRCA mutations and survival in breast cancer: an updated systematic review and meta-analysis. Oncotarget 7(43): 7011370127, 2016. PMID: 27659521. DOI: 10.18632/oncotarget.12158

32 Huang D, Sun W, Zhou Y, Li P, Chen F, Chen H, Xia D, Xu E, Lai M, Wu Y and Zhang H: Mutations of key driver genes in colorectal cancer progression and metastasis. Cancer Metastasis Rev 37(1): 173-187, 2018. PMID: 29322354. DOI: 10.1007/ s10555-017-9726-5

Received June 15, 2021

Revised July 19, 2021

Accepted July 22, 2021 